\title{
Biomechanics of running: An overview on gait cycle
}

\author{
Ekta Kapri a, ${ }^{*} \mathbb{D}$, Manju Mehta a, Kiran Singh a \\ a Department of Family Resource Management, CCSHAU, Hisar-125004, Haryana, India. \\ *Corresponding author Email: etikaprirai@gmail.com \\ DOI: https://doi.org/10.34256/ijpefs2131
}

Received: 20-05-2021, Revised: 03-07-2021; Accepted: 04-07-2021; Published: 05-07-2021

Abstract: This review article summarized the literature regarding running gait. It describes characteristics of running gait and running gait cycle, explains running anatomy in relation to lower and upper body mechanism; contribution of muscles, and joint running gait cycle. The concept of running kinematics and kinetics has described motion characteristics such as position, velocity, acceleration, and force applied during the running cycle. Running gait analysis techniques has discussed such as motion analysis, force plate analysis, and electromyography.

Keywords: Biomechanics, Running Analysis, Kinematics, Kinetics

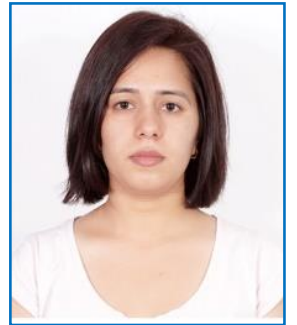

Ms. Ekta Kapri is a Ph.D. student in Family Resource management from the I.C. College of Home Science, CCSHAU, Hisar, Haryana. She is senior research fellow and currently working in the field of sports ergonomics. Her research work mainly focused on the field hockey, sports activity profile, anthropometry, fatigue analysis.

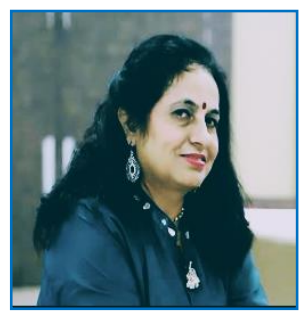

Dr. Manju Mehta received PhD in Resource Management. Currently, working as a Professor $\&$ Head of the department at the faculty of Family Resource management from the I.C. College of Home Science, CCSHAU, Hisar, Haryana. She is specialized in ergonomics and resource management. Author has over 110 research and scientific papers. She has teaching/ research experience of 28 years.

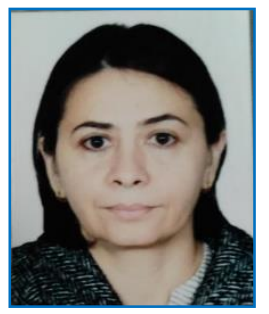

Dr. Kiran Singh is $\mathrm{PhD}$ and currently working as as Princple Scientist in the Department of Family Resource Management, I.C. College of Home Science, CCSHAU, Hisar, Haryana. She is specialized in Ergonomics, Gerontology and Women studies. She was the marathon runner and represented state in swimming and cross-country. She is sports coordinator of the college and also member in university sports committee.

\section{Introduction}

Sports activities and exercises are known to have a positive influence on individual's physical fitness, as well as reduce the incidence of obesity, cardiovascular diseases, and many other chronic health problems [1]. Running is one of the most popular sporting activities worldwide, for both the athletic and nonathletic people. The term "running" means a highintensity and inexpensive training in which a human's anatomical structure can be used. Over the past few decades, the attentiveness of people towards physical fitness has grown rapidly, but due to the time constraints, people have failed to take advantage of their athletic capabilities. Running discipline can be performed over a variety of distance and speeds, ranging from jogging to sprint and the most common of which are recreational running [2-5]. Recently it has become the most often used exercise to improve health. Even at relatively low doses ( 5 to 10 minutes per day), prolonged duration of running reduces the risk of death and makes it one of the healthiest activities [6]. Understanding biomechanics in the context of running can help to improve and analyze the technique of running and prevent injuries.

Running is a complex, coordinated process that depends on whole-body interaction, dividing the running cycle up into different phases can help us to understand the slight changes in the body segments during running [7-9]. Like any sports running also requires technique, and skill for improvement and 
active development of sportspersons. Similarly, the elementary understanding of principles and techniques of running biomechanics can be helpful to boost the performance of the runners and reduce susceptibility to injury. Biomechanics is a branch of biophysics that studies the human structure, motion, and movement technique. In sports, biomechanics, the technique is the dominant factor rather than the physical structure or physiological capacities, the laws of mechanics are applied to human movement in order to gain a greater understanding of athletic performance [7]. To examining the kinematics, biomechanics of running has been investigating (e.g. position, displacement, velocity, and acceleration) the joints and the body segments during the gait cycle, it also assesses the runner's muscles strength, their body flexibility, and balance; identify any deficiencies and highlight abnormality within their performing technique in repetitive sequence [10].

This article aims to understand the characteristics of running gait and gait cycle irrespective of the lower and upper body involvement and modern technology used to analyze the functional ability of an athlete.

\section{Methodology}

The recent and old both type of literature was used to understand the different sections of the article. Google Scholar, Scopus, research gate, PubMed, and online blogs and articles were used as the online sources, and books, sports journals, bulletins, and magazines were the offline sources. The following keyword has been used while searching the online literature like running, running biomechanics, gait cycle, type of gait, running kinetics and kinematics, and motion analysis. A total 64 articles were taken as a reference and used for the study.

\section{Characteristics of running gait}

Running velocity, stride and step length, stride and step frequency, running economy are some characters that influence an athlete running performance. Stride and step length are the parts of running velocity and running velocity is product of stride length and frequency. Stride length comprise two step lengths (Equation1), means it is distance between consecutive initial touchdown of the same foot (Figure1) and step length is distance between the heel contacts of one foot to another foot (Figure 1). Stride and step length depends on length of the leg, rang of motion of hip and strength of leg extension [10-15].

Stride length $=2 \times$ Step length $(\mathrm{m} / \mathrm{st}) \quad$ (Equation1)

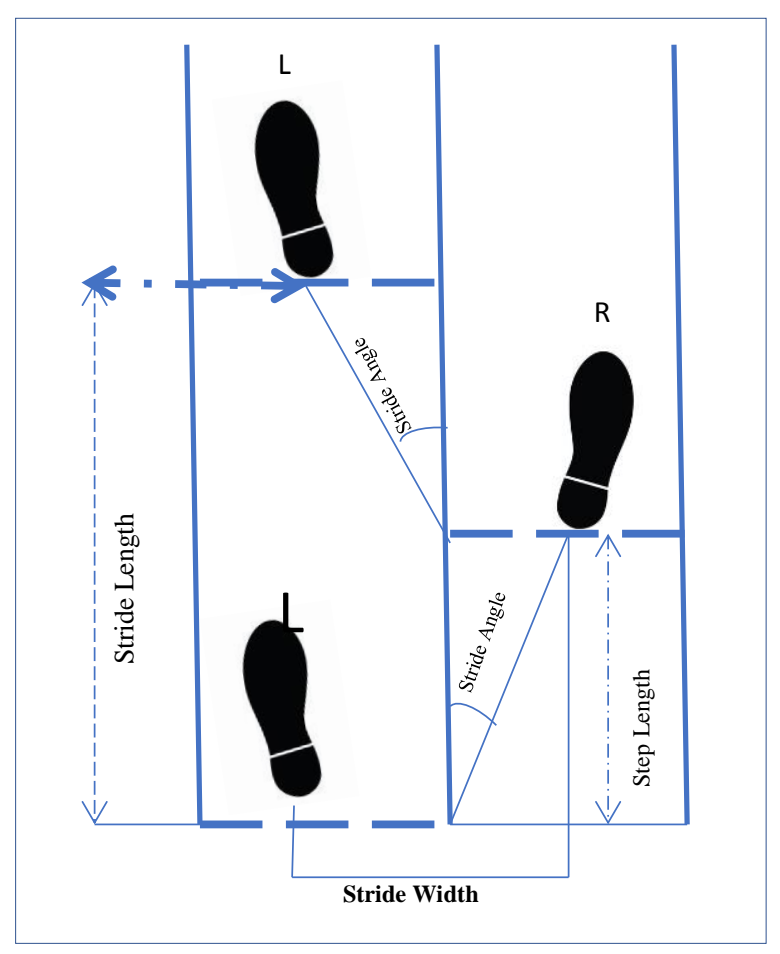

Figure 1. Stride length in a gait cycle

Stride length (SL) and stride frequency (SF) both are completely dependent on the leg length and range of movement at the hip joint and ratio (SL/SF) that varies with speed $\left(V_{x}\right)$ (Equation 2). Height of the athletes and leg length having direct but inverse relationship with stride frequency. At a given speed runners can voluntarily modify Stride frequency with running style [16].

$$
\mathrm{V}_{\mathrm{x}}=\mathrm{SL} / \mathrm{SF} \quad \text { (Equation 2) }
$$

Stride and step frequency is the rate of completing the stride and step. SF is simply a conversion of step time ( $\left.t_{\text {step }}\right)$; step time ( $\left.t_{\text {step }}\right)$ is addition of stance time ( $\left.t_{\text {stance }}\right)$ and flight time ( $\mathrm{t}_{\text {flight }}$ ) which can be calculated as SF $=60 /$ (tstance $+t_{\text {flight }}$ ) (Equation 3) where, $S F=60 / t_{\text {step }}$ and $t_{\text {step }}=t_{\text {stance }}+t_{\text {flight }}$ [17]. It's also depends on speed of muscle contraction and the skill of running. With increase the running speed stride length and frequency has also increased. With increase in running velocity swing phase increases and contact phase decreases and the point of initial contact has also changed [12-15]. Running economy can be good predictor of running velocity which measures energy utilization during running by assessing oxygen use at a given velocity [18, 19]. Through a process of self optimization experienced 
runners mostly adopted particular running style to utilize low energy [20, 21]. Human physiology, anthropometry, biomechanics, technique of training, age, gender, environmental conditions are some important factors contribute to determine running economy $[19,22,23]$. Therefore total running gait cycle have relation with all these running characteristics and change in these characteristics make changes in the running gait cycle.

\section{The Running Gait cycle}

Running is similar to walking in terms of locomotors activity. Every individual has different manner of movement on foot. Running is a type of gait in which, at the same time, at some point all feet are off the ground. Shultz (2015) describes gait as: "someone's manner of ambulation or locomotion, involves the total body" [24]. During the gait cycle, motion of the foot can be affected by the movement of the bones and joints in the lower extremity. In regard to the lower extremity, running gait can be divided into two phases - stance or support phase and swing or recovery phase, where one foot is on the ground (stance) and both feet are off the ground (swing) (Figure 2).
The running gait cycle begins when one foot comes in contact with the ground (beginning of the stance phase) and ends when the same foot contacts the ground again $[10,12]$. The stance phase starts at initial touch and ends at toe off; toe off marks the beginning of the swing phase of the cycle $[8,25]$.

Table 1 describes that stance phase, divided into two half: first half from initial touchdown to midstance, concerned with absorption (pronation) and second half from mid-stance to take-off, concerned with propulsion (supination); similarly, swing phase first half (initial swing) from take-off to mid swing and second half (terminal swing) from mid-swing to initial touch; therefore in running one cycle start from initial touch and end in initial touch, which is the first step of the next cycle $[10,12]$.

Stance phase cover $60 \%$ of the total cycle (Figure 2), which consists of two periods of doublelimb support (each 12\%) and one period of single-limb support (35\%); and a swing phase covers $40 \%$ of total running gait cycle (Figure 2) $[12,26]$. Toe-off is the midpoint of the one cycle and complete $50 \%$ of the cycle. During running, most of the forward force can be generated by the arm and leg swinging [27].

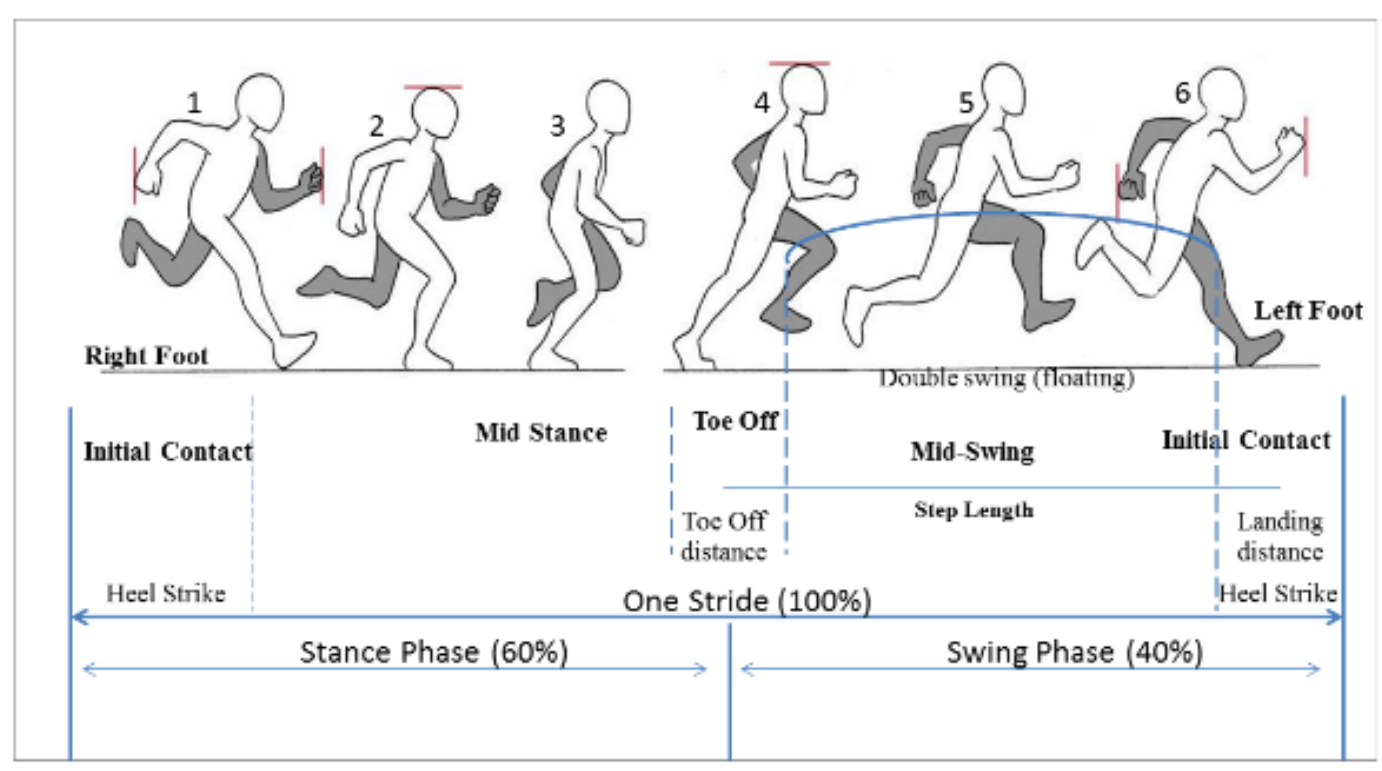

Figure 2. The gait cycle: Represents the different phases of the cycle

Table 1. Phases of gait cycle

\begin{tabular}{|l|l|l|l|}
\hline \multicolumn{2}{|l|}{ Stance Phase (Support) } & Swing Phase (Recovery) \\
\hline First Half & Second Half & First Half & Second Half \\
\hline $\begin{array}{l}\text { Initial touchdown - mid } \\
\text { stance (Pronation) }\end{array}$ & $\begin{array}{l}\text { Mid stance - toe off } \\
\text { (Supination) }\end{array}$ & $\begin{array}{l}\text { Toe off - mid swing (initial } \\
\text { swing) }\end{array}$ & $\begin{array}{l}\text { Mid swing - initial } \\
\text { touchdown } \\
\text { swing) }\end{array}$ \\
\hline
\end{tabular}


Therefore, the continuous nature of running gait from toe-off to toe-off, no certain point is assumed to be the beginning $[18,28,29,30]$.

\section{Running anatomy: lower and upper body mechanism}

Running biomechanics can be analyzed by upper and lower limb anatomy. In the running, upper body (head, arms and upper trunk) and lower body (lower trunk and legs) move in contrary directions in the longitudinal axis with contrary angular movement $[17,32]$. Almost all the muscle in the lower extremity is used in running. During running, the athlete's legs move forward quadriceps muscle (front thigh) bends the hip and straighten the knee. During forward movement hamstring (back thigh), soleus (inner calf) and gastrocnemius (outer calf) muscles, straighten the hip, bend the knee and extend-flex each foot during the gait cycle. Gluteal muscle (buttock) helps to extend the hip and stabilize the trunk. Hip extenders and hamstring muscles work together by extending hip during the first half of the stance and second half of swing $[29,33]$. In the whole gait cycle, foot and ankle joints move in all the planes with a predominant plane of motion known as "pronation" \& "supination", which cause obligate motion in the entire lower limb.

Initial contact during running eventuates with heel touch which requires neutral ankle dorsiflex. Tibia position enables this movement as maximum body weight is transferred to the stance leg. During the stance phase in running ankle dorsiflex is maximum, because the plantarflexed position and minimum duration of absorption period (pronation). Maximum ankle dorsi- and plantarflexion is depending on the speed of the athlete. In the first phase of the cycle knee does not extend but as the speed increases in the knee flexion increases approximately $45^{\circ}$ and hip flexes $30^{\circ}$. Hip movement from $10^{\circ}$ of flexion to extension during midstance but less extended while takeoff phase and knee extension to an average of $25^{\circ}$. In the early swing, hip extends $\left(10^{\circ}\right)$ and in mid-swing hip flexes $30^{\circ}$ [24, 29, 34]. Lower body muscles continue to produce ground reaction force or greater range of motion through muscle and joints, also coordinate with the upper extremities, and promoting efficient movement. In various phase of an athlete's leg action, hip are rotated back and forth in a horizontal plan. The rotator action of the hips induces contrary reaction in the athlete's upper body [35]. Running changes, the inclination of upper trunk ranging between $5^{\circ}$ to $7.5^{\circ}$ [36], and arm movement counteracting the rapid change in the trunks' angular moment which changes the body more gradually. Balance among the lower and upper body parts achieved by the opposite working of legs and arms, for example, right arm forward opposes the forward drive of the left leg. As the speed of locomotion increases, the elbows are flexed and the amplitude of the swing is enlarged to increase the angular momentum of the arm. In the first phase of running, during absorption (initial contact to midstance) the upper body produces propulsive force and during propulation (midstance to toe-off), the upper body produces an absorptive force $[8,35-$ 39]. In the second phase of running gait, if left knee creates anticlockwise momentum to counter balance this right arm and shoulder create a clockwise momentum to reduce rotational forces.

\section{Kinematics and kinetics of Running}

In the term, running kinematics is the description of motion characteristic such as position, velocity $\&$ acceleration of the lower extremities during a running cycle. It analyzes the gait pattern, change in the center of mass (COM) of body during locomotion, range of motion of various segments [40]. Kinematics analysis of running associated with different phases of the running like stride length and stride rate, change with speed, joint \& segment angle and movement of the trunk $\&$ arms. Stride length and stride rate (stride frequency) have relationship with speed and stride time; with increasing the stride length \& stride rate, stride time will decrease but speed (velocity) increases [41]. At the high speed range of motion, involvement of joint angle help to increase the speed and balance the body, example maximal thigh angles during hip flexion, prior to foot strike increase speed. Similarly increase in knee angle or knee flexion at foot strike and swing phase has been shown to increased running speed. The value in degrees of movement is depending on the athlete's level of training and speed [29]. Movement of trunk and arms while running balance the body movement, help to reduce overall energy expenditure and improve overall running system by helping to lift the body off the ground with each stride. Vertical oscillation of the center of mass (COM) has been decrease with increase running speed $[40,42]$. It can be shown when observing sagittal plan motion that COM is lowered as motion changes from walking to running to sprint. As running speed increases, pelvis and trunk tilt forward, but pelvic movement minimizes to conserve energy and maintain the efficiency in running [29]. 
Kinetics of the running is the study of applied force. Center of pressure method used for the assessment of force apply to the foot [15]. In the contact phase of running cycle, greatest stress application to the body known as ground reaction force (GRF). The greater the speed of running, greater the magnitude of vertical ground reaction force (VGRF). Ground reaction force (GRF) is determined by movement of the body and it is interesting to know to what extent movement of the various segments influence ground reaction can force (GRF) [40]. Hinrichs (1983) examined indirectly how the arms, legs, and trunk affect vertical ground reaction force (VGRF) during running by measuring the relative inertial force of the segment [43]. Kinetic and potential energy peak in mid-swing, center of mass (COM) fall toward the ground and as foot contact to the ground kinetic energy has lost. Energy for this movement is supplied by the active contraction of the muscles and the release of the elastic potential energy stored in the ligament and tendon [29].

\section{Running gait analysis and techniques}

Functional ability of a person varies according to sports and effective mobility of sportsmen is usually counted by the level of performance. Gait analysis of runners provides information about their running style and important to examine the biomechanical mobility of lower limb in dynamic movement [44]. Objective methods of running gait used to assess the spatial quantitative parameters such as step length, stride length, step width, foot angle, ground force, joint motion and joint rotation; all these parameters provide essential information about running style and depend on age, sex, anatomical characteristics, speed, endurance power, ability or state of training [45]. During running, the foot change velocities in short periods of time as it accelerates \& decelerates through the running cycle. Gait measurement systems helps to measure the stride characteristic and also understanding the internal and external force acting on body. Human gait analysis technological device can be classified as wearable sensor (WS), non wearable sensor (NWS) and hybrid system (combine both WS and NWS). WS system can be used outside the laboratory and record the data in open field situation. Different type of sensor attached on the several body parts like hand, legs, thigh or waist and these sensors record the signals that characterize the human gait [46]. In the contrast NWS are mostly used in the laboratory with controlled research facilities where subjects were instructed to follow the marked walkway. In the hybrid system WS and NWS both used by the researcher, example EMG sensor record the muscles activity and force platform used for measuring the stride length, frequency, GRF etc.

\subsection{Motion analysis technique}

The main goal of the human movement analysis is the acquisition of quantitative information about the mechanics of the musculoskeletal system while executing a motor task [47]. This technique captures the kinematic information of the movement of limbs by high- resolution cameras \& video recording device. Infrared transmitters (optical active marker) and retro reflective markers (optical passive marker) can be used along with the motion capture system [48]. Motion analysis technique is two types' marks based motion capture and mark less motion capture. Marker-based analysis is performed by retro-reflective markers on the body of the subjects. These retroreflector markers reflect light back to the source without scattering. Retro-reflective markers are used an array of light-emitting diodes (LEDs) mounted around the lens of each camera [49]. Marker-less human motion capture systems are proposed where conventional cameras can be utilized without the necessity of using special apparel or hardware [50]. In comparison to marker-based technique, marker-less motion capture system ensures an important reduction of the amount of time for setup preparation because no specialized operator is needed to place markers on the skin [51]. These motion analysis techniques require multiple camera viewpoints or 3-D sensors, but it can also be operated using a single-camera input without recourse to 3-D motion [52].

\subsection{Floor sensors}

Floor sensors are most commonly used in the biomechanical analysis of running. It used to measure steps, time elapsed in each position, heel and toe impact and generate prototype image of foot. There are two types of floor sensors: force platforms and pressure measurement systems. Pressure measurement systems quantifying the applied forces patterns under a foot vertically [53]. Muro-de-laHerran, etal (2014) described the pressure measurement system and found when the heel touches the floor and toe push off to take next step, during this time pressure may reach up to $120 \%-$ $150 \%$ of the body weight of individual [46]. Whereas Force plate generally used to measure the ground reaction force under the foot while running. It is based on the Newton's third law of motion: "for action there 
is an equal and opposite reaction". For gait analysis, force platforms fixed in the ground and record ground reaction force between the ground and the plantar surface of the foot; strain-gauge or piezoelectric transducers technique are the most widely used model in force platform (or a force plate) [49]. It helps to quantify balance, gait and ground reaction force (which can be vertical, horizontal and rotator plane during stance phase). In the context of analyzing the gait cycle force plates are determining the heel-strike and toe-off phases by recording the ground reaction forces [49]. Vertical load, horizontal shear, joint torques \& center of pressure determination are the most useful data from the force plate analysis.

\subsection{Electromyography (EMG)}

Electromyography is a wearable sensor and notable technique used in biomechanics to record electrical manifestation of the contracting muscle in the dynamic activity. Oscilloscopes are used in order to monitor the raw EMG signals. Biofeedback, force generation by voluntary or involuntary muscle, muscle tensions and fatigue within a muscle measured by noninvasively (surface electrodes) or invasively (wire or needle electrodes) $[46,49,54,55]$. Measured and recorded analog signals amplified into the yield format. Furthermore, these signals help in recording of muscles contraction frequency and intensity, kinematic plots of joint angular motion and changes in passive muscletendon properties $[45,46]$. It also concerned with contraction of muscles during the simple movement such as flexion, extension, abduction, adduction, rotation, inversion, eversion and combination of these movements [56]. EMG of lower extremities assessed by phasic activity presented by different muscle groups such as flexors \& extensors of hip and knee and ankle dorsiflexors \& planter flexors. Subbu, Weiler, and Whyte, (2015) correlate EMG activity with running velocity and muscle fatigue, found change in the muscles function always the consequence of prolonged, dynamic activity [57].

\subsection{Inertial Sensors}

Inertial sensors or inertial measurement units (IMUs) is electronic devices, used with the combination of accelerometers and gyroscopes. It is based on inertial/magnetic sensor modules in which micro electro-mechanical systems (MEMS) sensors are used, which make it possible to track orientation in real time [49]. Sant'Anna et al. (2013) developed a system with inertial sensors to quantify gait symmetry and gait normality with subject's velocity, acceleration, orientation, and gravitational forces [58]. In the inertial sensor, accelerometers basically used to measure the acceleration of a body in proportion to the net force acting on the body during running. Accelerometers work on spring mass system theory, where small mass used in order to calculate the applied acceleration. Uni-axial, bi-axial, and triaxial accelerometers records acceleration in single, double and three orthogonal axes respectively [49, 59]. Gyroscopes are based on the property of inertia, consisting of a vibrating element merged with a sensing element and used as a reference to detect changes in direction. It recorded data of angular velocity (joint angle) without being affected by gravity and linear acceleration $[46,49]$. To estimate the orientation of the different segment of the body sensor placed to the major segments. Example to track gait movements, integrated sensors placed on each ankle position along with a body sensor positioned near the cervical vertebra to monitor body posture. stride index has used with inertial measurement units (IMUs) to assess complete time intervals from foot strike until the complete landing of the whole foot on the ground $[60,61]$. Shih et al. found high positive correlation between IMU and motion system and record the changes of the foot during intense running [62]. This sensor also useful in diseases where gait disorders are a symptom like Parkinson's [63].

\section{Conclusion}

This article summarized that running biomechanics has improved immensely form the past few decades. Biomechanical study on running improve knowledge about gait type, human posture (anatomy) while running and underlying cause of movement to identify the difference among individuals performing speed. Primary motives of the biomechanical evaluation of running are to identify the factors essential the performance and it may also indeed enable a precise quantification of motor techniques with a purpose to optimize, develop human performance. Runner's body parts contribute in the movement, balance and stability of body during running and also determine the running speed. Running gait analysis technique helps to record the kinetic and kinematics aspect which can provide the data about speed, locomotion of the joint, magnitude and direction of external force acting on the limbs during different phase of running. 
Gait analysis technique measure the functional ability of an athlete quantitative parameters such as step and stride length, foot angle, ground force, joint motion and rotation. These parameters have recorded by the wearable and non-wearable sensors such as motion analysis technique, floor sensors, electromyography and inertial sensor.

\section{References}

[1] R.N. Van Gent, D. Siem, M. Van Middelkoop, A.G. Van Os, S. M. A. Bierma-Zeinstra, B.W. Koes, Incidence and Determinants of Lower Extremity Running Injuries in Long Distance Runners: A Systematic Review, British Journal of Sports Medicine, 41(8), (2007) 469-480. [DOI] | [PubMed]

[2] C.W. Chan, A. Rudins, Foot Biomechanics during Walking and Running, In Mayo Clinic Proceedings, 69(5) (1994) 448-461. [DOI]

[3] B. Knechtle, P. Knechtle, I. Schulze, \& G. Kohler, Upper Arm Circumference Is Associated with Race Performance In Ultra-Endurance Runners. British Journal of Sports Medicine, 42(4), (2008) 295-299. [DOI] I [PubMed]

[4] R. Dermack, Long-Distance Running: An Investigation into Its Impact on Human Health (2015). Access from https://Pitjournal.Unc.Edu/Article/LongDistance-Running-Investigation-Its-ImpactHuman-Health

[5] L. Vitez, P. Zupet, V. Zadnik, M. Drobnič, Running Injuries in the Participants of Ljubljana Marathon, Slovenian Journal of Public Health, 56(4), (2017) 196-202. [DOI] । [PubMed]

[6] K.R. Williams, Biomechanics of Running, Exercise and Sport Science Review, 13, (1985) 389-441.

[7] D. Knudson, Fundamentals of Biomechanics, Springer Science \& Business Media (2007).

[8] M. Phillips, Introduction to Running Biomechanics, (2013), Retrieved from https://Runnersconnect.Net/Running-

Biomechanics/ On 13 May 2021.

[9] R.B. Souza, An Evidence-Based Videotaped Running Biomechanics Analysis, Physical Medicine and Rehabilitation Clinics, 27(1), (2016) 217-236. [DOI] I [PubMed]

[10] C.F. Bridgman, Biomechanical Evaluation of Distance Running During Training and Competition, Doctoral Dissertation, University
Of Salford, (2015). Available on:

http://usir.salford.ac.uk/id/eprint/34545/

[11] S. Ounpuu, The Biomechanics of Running: A Kinematic and Kinetic Analysis, Instructional Course Lectures, 39, (1990) 305-318. [PubMed]

[12] S.A. Dugan, K.P. Bhat, Biomechanics and Analysis of Running Gait, Physical Medicine and Rehabilitation Clinics, 16(3), (2005) 603621. [DOI] | [PubMed]

[13] N. Hamilton, K. Luttgens, The Lower Extremity: The Knee, Ankle and Foot. Kinesiology, Scientific Basis of Human Motion, 10, (2002) 182-192.

[14] R.S. Adelaar, The Practical Biomechanics of Running, The American Journal of Sports Medicine, 14(6), (1986) 497-500. [DOI] । [PubMed]

[15] P.R. Cavanagh, The Biomechanics of Lower Extremity Action in Distance Running, Foot \& Ankle, 7(4), (1987) 197-217. [DOI] I [PubMed]

[16] I.S. Moore, Is There an Economical Running Technique? A Review of Modifiable Biomechanical Factors Affecting Running Economy, Sports Medicine, 46(6), (2016) 793807. [DOI] | [PubMed]

[17] B.T. Van Oeveren, C.J. de Ruiter, P.J. Beek, J.H. Van Dieën, The biomechanics of running and running styles: a synthesis, Sports Biomechanics, (2021) 1-39. [DOI] | [PubMed]

[18] T. Anderson, Biomechanics and Running Economy, Sports Medicine, 22(2), (1996) 7689. [DOI] | [PubMed]

[19] P.U. Saunders, D.B. Pyne, R.D. Telford, J.A. Hawley, Factors Affecting Running Economy in Trained Distance Runners, Sports Medicine, 34(7), (2004) 465-485. [DOI] I [PubMed]

[20] P.R. Cavanagh, K.R. Williams, The Effect of Stride Length Variation on Oxygen Uptake during Distance Running, Medicine and Science in Sports and Exercise, 14(1), (1982) 30-35. [DOI]

[21] I.S. Moore, A.M. Jones, S.J. Dixon, Mechanisms for Improved Running Economy in Beginner Runners, Medicine and Science in Sports and Exercise, 44(9), (2012) 1756-1763. [DOI] | [PubMed]

[22] G. Crowther, Tips on Maximizing Your Running Economy. Northwest Runner Magazine (2001), USA.

[23] T. Noakes, Lore of Running, Human Kinetics, (2003) 29-47. 
[24] S.J. Shultz, P.A. Houglum, D.H. Perrin, Examination of musculoskeletal injuries, Human Kinetics, (2015) 55-60.

[25] Tekscan. The Gait Cycle: Phases, Parameters to Evaluate \& Technology, (2019), Retrieved from

https://Www.Tekscan.Com/Blog/Medical/GaitCycle-Phases-Parameters-Evaluate-Technology On 8 May 2021.

[26] R.M. Buschbacher, N.D. Prahlow, S.J. Dave, (Eds.), Sports Medicine and Rehabilitation: A Sport-Specific Approach, Lippincott Williams \& Wilkins (2008), uSA.

[27] R.A. Mann, J. Hagy, Biomechanics of walking, running, and sprinting, Am J Sports Med,8(5) (1980) 345-350. [DOI] I [PubMed]

[28] T.L. Nicola, D.J. Jewison, The Anatomy and Biomechanics of Running, Clinics in Sports Medicine, 31(2), (2012) 187-201. [DOI] । [PubMed]

[29] T.F. Novacheck, The Biomechanics of Running, Gait \& Posture, 7(1), (1998) 77-95. [DOI]

[30] R. Bartlett, Introduction to sports biomechanics: Analyzing human movement patterns, Routledge, (2007) 15-16. [DOI] । [PubMed]

[31] T. F. Novacheck, Walking, running, and sprinting: a three-dimensional analysis of kinematics and kinetics, Instructional course lectures, 44, (1995) 497-506. [PubMed]

[32] S.R. Hamner, S.L Delp, Muscle contributions to fore-aft and vertical body mass center accelerations over a range of running speeds, Journal of biomechanics, 46(4), (2013) 780787. [DOI] | [PubMed]

[33] J. Kelly. How Leg Workouts For Runners Work (2020), Access From https://Adventure.Howstuffworks.Com/Outdoo r-Activities/Running/Training/Leg-WorkoutsFor-Runners1.Htm, On 15 May 2021

[34] J.K. Loudon, M. Swift, S. Bell, The Clinical Orthopedic Assessment Guide, Human Kinetics, (2008), USA.

[35] J. Hay, The Biomechanics of Sports Techniques, (1978) 411-412, Prentice-Hall, USA.

[36] S.J. Preece, D. Mason, C. Bramah, The Coordinated Movement of the Spine and Pelvis during Running, Human Movement Science, 45, (2016) 110-118. [DOI] | [PubMed]
[37] F. Bosch, K. Klomp, Biomechanics and exercise physiology applied in practice, (2005), Elsevier Churchill Livingstone, USA.

[38] J.W. Bunn, Scientific Principles of Coaching, (1972) 95, Prentice Hall, USA.

[39] B.J. Hopper, The Mechanics of Arm Action in Running, Track Technique, 17, (1964) 520522.

[40] R.L. Terjung, Exercise and Sports Science Review, American College of Sports Medicine Series, 13 (1985) 389-431.

[41] B.C. Elliott, B.A. Blanksby, Optimal Stride Length Considerations for Male and Female Recreational Runners, British Journal of Sports Medicine, 13(1), (1979) 15-18. [DOI]

[42] M. Lindsay, How Arm Swing Affects Your Running Efficiency, (2018) Access From https://Blog.Mapmyrun.Com/How-Arm-SwingAffects-Your-Running-Efficiency/ On 15 May 2021.

[43] R.N. Hinrichs, Upper Extremity Function in Running, Phd Thesis, Pennsylvania State University, (1983).

[44] S. Langer, A Practical Manual of Clinical Electrodynography, Langer Foundation for Biomechanics and Sports Medicine Research, (1989).

[45] C. Frigo, M. Rabuffetti, D. C. Kerrigan, L. C. Deming, \& A. Pedotti, Functionally Oriented and Clinically Feasible Quantitative Gait Analysis Method, Medical and Biological Engineering and Computing, 36(2), (1998) 179-185. [DOI] | [PubMed]

[46] A. Muro-De-La-Herran, B. Garcia-Zapirain, A. Mendez-Zorrilla, Gait Analysis Methods: An Overview of Wearable And Non-Wearable Systems, Highlighting Clinical Applications, Sensors, 14(2), (2014) 3362-3394. [DOI] । [PubMed]

[47] A.Cappozzo, U. Della Croce, A. Leardini, \& L. Chiari, Human Movement Analysis Using Stereophotogrammetry: Part 1: Theoretical Background. Gait \& Posture, 21(2), (2005) 186-196. [DOI] | [PubMed]

[48] E. Van Der Kruk, \& M. M. Reijne, Accuracy of Human Motion Capture Systems for Sport Applications; State-Of-The-Art Review, European Journal of Sport Science, 18(6), (2018) 806-819. [DOI] I [PubMed]

[49] E. Surer, A. Kose, Methods and Technologies for Gait Analysis, In Computer Analysis of 
Human Behavior, Springer, London, (2011) 105-123. [DOI]

[50] J. Deutscher, A. Blake, I. Reid, Articulated Body Motion Capture by Annealed Particle Filtering, In Proceedings IEEE Conference on Computer Vision and Pattern Recognition. CVPR 2000 (Cat. No. PR00662) (2), (2000) 126-133. [PubMed]

[51] S. Corazza, L. Muendermann, A.M. Chaudhari, T. Demattio, C. Cobelli, T.P Andriacchi, A Markerless Motion Capture System to Study Musculoskeletal Biomechanics: Visual Hull and Simulated Annealing Approach, Annals of Biomedical Engineering, 34(6), (2006) 10191029. [DOI]

[52] T.J. Cham, J.M. Rehg, A Multiple Hypothesis Approach to Figure Tracking. In Proceedings. 1999 IEEE Computer Society Conference on Computer Vision and Pattern Recognition (Cat. No PR00149) (2), (1999) 239-244. [DOI]

[53] D.G.E. Robertson, G.E. Caldwell, J. Hamill, G. Kamen, S. Whittlesey, Research methods in biomechanics, Human kinetics, (2013).

[54] G.L. Soderberg, T.M. Cook, Electromyography in Biomechanics, Physical Therapy, 64(12) (1984) 1813-1820. [DOI] | [PubMed]

[55] J.P. Clarys, J. Cabri, (1993). Electromyography and the Study of Sports Movements: A Review, Journal of Sports Sciences, 11(5), 379-448. [DOI]

[56] G.W. Hermann, An Electromyographic Study of Selected Muscles Involved in the Shot Put, Research Quarterly, American Association for Health, Physical Education and Recreation, 33(1), (1962) 85-93. [DOI]

[57] R. Subbu, R. Weiler, G. Whyte, The practical use of surface electromyography during running: does the evidence support the hype? A narrative review, BMJ open sport \& exercise medicine, 1(1), (2015) e000026. [DOI] । [PubMed]

[58] A. Sant'Anna, N. Wickström, H. Eklund, R. Zügner, \& R. Tranberg, Assessment of gait symmetry and gait normality using inertial sensors: in-lab and in-situ evaluation, In International Joint Conference on Biomedical Engineering Systems and Technologies, Springer, Berlin, Heidelberg, (2012) 239-254. [DOI]

[59] M.J. Mathie, B.G. Celler, N.H. Lovell, A.C.F. Coster, Classification of basic daily movements using a triaxial accelerometer, Medical \&
Biological Engineering \& Computing, 42, (2004) 679-687. [DOI] I [PubMed]

[60] A. Tay, S.C. Yen, J.Z. Li, W.W. Lee, K. Yogaprakash, C. Chung, S. Liew, B. David, W.L. Au, Real-Time Gait Monitoring For Parkinson Disease, In Proceedings of 2013 10th IEEE International Conference on Control and Automation (ICCA), Hangzhou, China, (2013) 1796-1801.

[61] T.Y. Shiang, T.Y. Hsieh, Y.S. Lee, C.C. Wu, M.C. Yu, C.H. Mei, I.H. Tai, Determine the foot strike pattern using inertial sensors, Journal of Sensors, (2016). [DOI]

[62] Y. Shih, K.L. Lin, T.Y. Shiang, Is the foot striking pattern more important than barefoot or shod conditions in running? Gait \& posture, 38(3), (2013) 490-494. [DOI] I [PubMed]

[63] A. Salarian, H. Russmann, F.J.G. Vingerhoets, C. Dehollaini, Y. Blanc, P.R. Burkhard, K. Aminian, Gait Assessment in Parkinson's disease: Toward an Ambulatory System for Long-Term Monitoring, IEEE Transactions on Biomedical Engineering, 51, (2004) 14341443. [DOI] | [PubMed]

\section{Funding}

No funding was received for conducting this study.

\section{Authors Contribution}

All the authors equally contributed to this work and approved of the final version of this manuscript.

\section{Conflict of interest}

The Authors have no conflicts of interest to declare that they are relevant to the content of this article.

\section{Does this article screened for similarity}

Yes

\section{About The License}

(c) The Author(s) 2021. The text of this article is open access and licensed under a Creative Commons Attribution 4.0 International License 